\title{
HOMOFOBIA EN LA ESCUELA: PSICOLOGÍA AFIRMATIVA GAY EN EL CONTEXTO EDUCATIVO
}

\author{
Begoña Sánchez Torrejón \\ Universidad de Cádiz \\ begonia.sanchez@uca.es \\ Cristobal Rivera Mera \\ Universidad de Cádiz \\ observatorio.diversaci@uca.es
}

Recepción Artículo: 13 octubre 2021

Admisión Evaluación: 13 octubre 2021

Informe Evaluador 1: 13 octubre 2021

Informe Evaluador 2: 14 octubre 2021

Aprobación Publicación: 14 octubre 2021

\section{RESUMEN}

En los últimos años ha aumentado significativamente el bullying homofóbico en los centros escolares. El presente artículo surge de la necesidad de escuchar y visibilizar las experiencias vividas por un alumno gay a lo largo de las diversas etapas educativas. La psicología afirmativa gay ofrece un enfoque de conocimiento y comprensión de las realidades y problemáticas concretas de las personas homosexuales. El objetivo de este artículo es analizar las vivencias homofóbicas que se han detectado en el acompañamiento terapéutico de un chico homosexual a lo largo de los diferentes ciclos educativos. Hemos utilizado la metodología biográfica-narrativa para dialogar con nuestro informante y acercarnos a una realidad compleja pero necesaria de abordar. Concretamente el instrumento empleado ha sido la entrevista semiestructurada. Entre los resultados obtenidos más destacados se refleja la necesidad de sensibilización y formación en la comunidad educativa sobre las diversidades sexo-genéricas desde una perspectiva inclusiva. A su vez, se pone de manifiesto la carencia de herramientas del profesorado para prevenir, detectar y abordar los casos de bullying homofóbico y promover la inclusión de la diversidad sexogenérica en los centros escolares.

Palabras clave: educación; homofobia; inclusión; psicología afirmativa

\section{ABSTRACT}

Homophobia at school: gay affirmative psychology in the educational context. Bullying for homophobic reasons has increased significantly in schools over the last years. This article responds to the necessity of acknowledging and raising awareness towards the experiences that a gay student faces throught diferent educational stages. The Gay affirmative psychotherapy focuses on understanding and comprehending the specific issues and realities of homosexual people. The aim of this paper is to anlyse the homofobic experiences that have 


\section{HOMOFOBIA EN LA ESCUELA: PSICOLOGÍA AFIRMATIVA GAY EN EL CONTEXTO EDUCATIVO}

been identified during the thereapeutic process of a gay person over the course of the diferent educational stages. We have used a biographic- narrative method in order to conduct a dialogue with our informer and come closer to a complex reality that needs to be addressed. The specific approach used for this purpose is the semi-structured interview. The most significant results show the necessity of understanding and raising awareness to sex and gender diversity from an inclusive perspective in the education community. The article also brings out the lack of tools teachers have when it comes to preventing, identifying and addressing bullying situations for homofobic reasons, as well as promoting the inclusion of sex and gender diversity within schools.

Keywords: education; homofobia; inclusión; affirmative psychology

\section{INTRODUCCIÓN}

La escuela es uno de los espacios donde las personas con orientaciones e identidades no normativas sufren mayor rechazo, discriminación y violencia (Martxeta y Etxeberria, 2014). En la socialización de la infancia y de la adolescencia, los centros escolares son elementos fundamentales en los procesos de la construcción de la identidad sexo-genérica (Berná, Cascone y Platero, 2012). La institución escolar es la primera institución política, independiente del ámbito familiar, en la que el alumnado está subordinado a la taxonomía binaria del género a través de la exigencia constante de los discursos impuestos desde la heteronormatividad. No podemos obviar, que los centros escolares potencian y valoran la reproducción heteronormativa desde las primeras etapas educativas de los códigos de identidad de género y de identidad sexual, al mismo tiempo que vigilan, castigando y patologizando, toda forma de disidencia del modelo hegemónico.

El espacio escolar es un agente clave en la socialización del género y la identidad, transmitiendo patrones y modulando la orientación e identidad sexual con los discursos que transmite y perpetúa, convirtiéndose en un sutil instrumento de represión (Foucault, 1976). Por todo ello, como señala Sánchez "la heteronormatividad que se trasmiten en los centros escolares impuestas por el modelo hegemónico comienzan a hacerse presentes desde temprana edad, repercuten directamente sobre la forma en la que las niñas y los niños se autoperciben y cómo ven a los demás" (2021, p.254-255).

Para comenzar a comprender la problemática social del bullying homofóbico, es necesario aclarar qué entendemos por este término. Con los terminos 'acoso escolar homofobico' o 'bullying homofobico' nos referimos:

aquellos comportamientos violentos por los que un alumno 0 alumna se expone y/o queda expuesto repetidamente a la exclusion, aislamiento, amenaza, insultos y agresiones por parte de sus iguales, una 0 varias personas que estan en su entorno mas proximo, en una relacion desigual de poder, donde los agresores o "bullies" se sirven de la homofobia, el sexismo, y los valores asociados al heterosexismo.(Platero y Gómez, 2007, p 14)

Autores como Agustín lo definen como: "Cualquier tipo de agresión verbal, física o social entre estudiantes que: bien está destinada a atacar a determinadas personas por su orientación sexual real o percibida o la de algún familiar 0 amigo" (2009, p.80). Además, el autor puntualiza que entre sus características se encuentran la reiteración de las conductas, la intencionalidad de ejercer daño tanto psicológico como físico y el desequilibrio de poder que tiene la víctima. Algunos autores señalan características propias de este tipo de bullying como son la invisibilidad de la diversidad sexo-genérica en las aulas, el contagio del estigma, el horizonte de la injuria, a normalización de las prácticas discriminatorias y la falta de redes de apoyo (Carrer y Cifuentes, 2019; Pichardo, 2012).

Diferentes autores como Blais, Gervias y Hebert (2014), postulan que el alumnado no heteronormativo tiene más riesgo de sufrir acoso escolar que el alumnado no perteneciente a este colectivo. Meyer (2003), especifica que es bastante más alto el riesgo para el alumnado gay de sufrir acoso y convertirse en víctimas del acoso escolar homofóbico, incluso más que las víctimas que sufren acoso escolar. El bullying homofóbico es un serio problema, que no podemos posponer en las agendas educativas, ya que está presente en nuestras aulas escolares desde las primeras etapas educativas. Rivers (2004), detectó en una investigación realizada a 119 personas entrevistadas que habían sufrido acoso homofóbico, que la edad media en la que se inicia el acoso escolar homofóbico era de 10,5 años. 
Varios estudios realizados (COGAM, 2016; Pichardo et al. 2015), reflejan aspectos preocupante con respecto al bullying homofóbico en los centros escolares, tales como la invisibilidad de las diversidades sexo-genéricas en el sistema educativo, la percepción negativa y llena de estereotipos homofóbicos, diversos comportamientos discriminatorios hacia el alumnado no heteronormativo, y, que aún sigue siendo un tema tabú en el ámbito escolar.

En esta línea, es interesante resaltar que una de las consecuencias directas en el ámbito educativo del bullying homofóbico son el fracaso y abandono escolar entre el alumnado no heterosexual, lo que incide directamente de manera negativa en la capacidad de estas personas para adaptarse adecuadamente a la escuela, al ámbito laboral y llegar a desarrollarse como adultos sanos (Takács, 2006). Los efectos del bullying homofóbico pueden llevar al abandono del centro escolar por parte del alumnado acosado, a la desmotivación académica, ansiedad e incluso a tener la víctima comportamientos de autolesión (Hadler, 2012; Garrido y Morales, 2014). Además, las experiencias de bullying homofóbico sufridas en la escuela según Quiceno et al. (2013), se relacionan con la depresión, la autoestima baja, Ilegando a provocar el abuso de sustancias, el aislamiento social y la tentativa de suicidio e incluso el suicidio.

A este respecto, como se refleja en las diversas investigaciones sobre el bullying en el contexto escolar por motivo de orientación afectivo-sexual, este es una fuente de estrés que influye de manera directa en la salud mental de las víctimas que lo sufren. A tenor de lo expuesto, consideramos necesario el acompañamiento psicológico a través de la psicología afirmativa gay.

La Psicología Afirmativa Gay surge como un enfoque que considera la homosexualidad tan normal como la heterosexualidad pero conociendo y respetando la realidad de las personas homosexuales, que difieren en gran medida de las personas heteronormativas en diversos aspectos, entre lo que estarían: la formación de relaciones entre personas del mismo sexo, los procesos de desarrollo de la sexualidad, la homofobia y discriminación sufridas, las cuestiones relativas a la maternidad y paternidad (Borges, 2009).

Por lo tanto la Psicología Afirmativa Gay surge en contraposición de las terapias de "conversión", las cuales están prohibidas debido a diversos motivos entre los cuales se encuentran: atentar contra la dignidad de las personas y el trato denigrante contra los seres humanos. Uno de los objetivos de la Psicología Afirmativa Gay a su vez, es ofrecer a las personas homosexuales la posibilidad de vivir en paz consigo mismas teniendo en cuenta que han de convivir en un contexto homófobo (Ardila, 2007).

Ya en 1973 la Asociación Americana de Psicología (American Psychology Association, APA) eliminó la homosexualidad como enfermedad mental. No mucho más tarde, en 1975, determinó que: "la homosexualidad como tal no implica daño alguno en el juicio de la persona, su estabilidad, su confiabilidad o sus capacidades generales sociales y vocacionales". Reconociendo a su vez la situación específica de las personas homosexuales, poniendo en valor el esfuerzo que realizan los profesionales para la mejor comprensión del estigma y las diferentes manifestaciones y consecuencias que puede tener en la vida de las personas no heteronormativas.

Más recientemente, en 2020, la APA define la homosexualidad como una atracción emocional, romántica 0 afectiva duradera hacia personas del mismo sexo. Definiendo la identidad de género como el sentido psicológico de ser hombre o mujer. Y define el rol social del sexo como el respeto de las normas culturales de conducta femenina y masculina. Más concretamente, la división 44 está comprometida con el avance en la justicia social en todas sus actividades. La sociedad ha de celebrar la diversidad de personas lesbianas, gais, bisexuales, transgéneros, personas no binarias y queer y reconoce la importancia de la diversidad que genera también la interacción de las diferentes dimensiones del ser humano entre las que se enumeran de forma no excluyente: raza, etnia, capacidades, ciudadanía, estado salud, idioma, nacionalidad, religión y clase social. Esta misma división ha confeccionado listas de psicoterapeutas que están especializados en psicoterapia con personas LGTBIQA+ con Ios problemas específicos: parejas, familia, homofobia interiorizada, etc.

En definitiva la Psicología Afirmativa Gay trabaja basándose en las evidencias existentes que afirman que las personas LGTBIA+ tienen mayor riesgo de padecer problemas de salud física y salud mental (Meyer, 2001). Son 


\section{HOMOFOBIA EN LA ESCUELA: PSICOLOGÍA AFIRMATIVA GAY EN EL CONTEXTO EDUCATIVO}

muchos los estudios que las personas que sufren bullying desarrollan trastornos de estrés postraumático como consecuencia de recordar las experiencias de acoso sufridas en la etapa escolar (Rivers, 2004; Rivers et al., 2006).

Además de la sintomatología anteriormente descrita, las investigaciones que han recogido la experiencia victimizante con jóvenes lesbianas, gais y bisexuales como consecuencia de su orientación afectivo-sexual, han descrito la existencia de correlación entre su orientación afectivo-sexual y la presencia de baja autoestima, depresión, ansiedad, abuso de sustancias, tentativa de suicidio e incluso suicidio consumado (D’Augelli, Pilkington y Hershberger, 2002; Espelage, Aragon, y Birkett, 2008, Martxueta y Etxeberria, 2014).

Es por ello que consideramos necesario el análisis y la comprensión de las vivencias de acoso escolar desde la Psicología Afirmativa Gay, ya que desde este enfoque no solo se respeta y celebra la diversidad, sino que ofrece la oportunidad de eliminar mediante psicoterapia los efectos negativos de la homofobia sufrida. De igual forma posibilita el poder actuar e intervenir en el contexto educativo y eliminar los comportamientos, actitudes y creencias homófobas.

\section{OBJETIVOS DE LA INVESTIGACIÓN}

El principal objetivo del presente artículo es analizar las vivencias homófobas de un chico homosexual en las diferentes etapas educativas.

Es por ello que planteamos los siguientes objetivos específicos:

Conocer las diferentes vivencias traumáticas en relación a la homosexualidad en el contexto educativo.

Detectar la homofobia interiorizada que ha podido desarrollar como consecuencia de dichas vivencias.

Observar las dificultades en el desarrollo de las habilidades sociales durante la infancia y adolescencia.

Conocer el desarrollo de la identidad afectivo-sexual a lo largo de las diferentes etapas educativas.

Promover la capacidad de resiliencia en un contexto homófobo.

\section{MUESTRA Y/O PARTICIPANTES}

Respecto al informante que ha participado en nuestro estudio, se trata de un chico de 18 años que vive en la provincia de Málaga. Actualmente acaba de finalizar $2^{\circ}$ de bachillerato y se dispone a comenzar la etapa universitaria en Barcelona. Durante su etapa de primaria realizó sus estudios en un colegio público y en secundaria en dos centros educativos; en primer lugar un colegio privado y posteriormente en un colegio público. Todos ellos en la provincia de Málaga. En todo momento, se ha mantenido el anonimato y el informante ha sido previamente informado de los diversos pasos llevados a cabo en la investigación. La toma de información se realizó en tres días, con una duración de una hora y media cada una de ellas.

No podemos olvidar las conductas éticas en el ejercicio de la investigación científica y en el uso del conocimiento producido. Autores como Bromseth postulan: "Ética y metodología son dos aspectos relacionados con consecuencias mutuas: un determinado punto de vista ético delimita aproximaciones metodológicas, y la elección de un determinado modo de resolver las preguntas de investigación implica una perspectiva ética (2002, p.35).

Entre los principios éticos a tener en cuenta tenemos el consentimiento informado, es un principio básico garante de la autonomía y del derecho a la privacidad de los datos de la persona informante. Este consiste en un proceso encaminado a que la persona participante conozca los riesgos, los beneficios y las consecuencias que se puedan presentar durante el desarrollo de la investigación en la cual participa (Vargas et al., 2007). Las personas que participan en una investigación deben ser conocedoras de todo el proceso "estas personas deben recibir información acerca de los objetivos de la investigación, el tipo de participación demandada o esperada, así como el uso que se hará de los resultados obtenidos" (Abad, 2015, p.105). A través del diálogo con el informante de esta investigación, hemos consensuado la firma del consentimiento informado de sus datos, facilitando toda la información necesaria para la toma de una decisión reflexionada. 
Además, debemos respetar los principios de la confidencialidad de la información obtenida y del anonimato de las personas investigadas. La confidencialidad de los datos incluye mantener la confidencialidad de los registros e información recolectada; y garantizar que las personas que tienen acceso a este tipo de información, también aseguren la confidencialidad (Wiles et al., 2006).

\section{METODOLOGÍA Y/O INSTRUMENTOS UTILIZADOS}

En la presente investigación utilizaremos la metodología biográfica-narrativa, esta nos abre a un itinerario caleidoscópico, ofreciéndonos la posibilidad de comprender una realidad compleja cómo es la homofobia en la educación, desde la propia mirada y sentir de un alumno gay a lo largo de las diversas etapas educativas. Uno de los aspectos a destacar de la investigación biográfica-narrativa, es que permite indagar e interpretar el mundo subjetivo de las personas informantes, exponiendo su modo de comprender y conocer la realidad, siendo protagonistas de su propia historia, dando sentido a las acciones y valores que la definen como grupo cultural (Boza et al., 2010).

Situamos a nuestra informante como el principal protagonista de la investigación, «solo convirtiéndolos en actores y actrices de sus propias vidas se podrá comprender y atribuir significado a los acontecimientos y situaciones vividas en el contexto de estudio» (Massot, 2003, p. 278). Por tanto, es un relato subjetivo, siendo esta una de sus principales características positivas, ya que el individuo puede analizar y comprender cómo determinadas situaciones o hechos puntuales en su vida han marcado su trayectoria académica y en definitiva, social.

Las historias de vida son un proceso de investigación que parten de una interrogante y, en este estudio, hemos utilizado esta metodología para conocer de primera mano, la percepción de nuestro informante, como señala Pujadas (2000, p. 141):

La elección del informante biográfico requiere de la adecuación del perfil de la persona, como estudio de caso, al tipo de testimonio que se adapte a los objetivos de nuestra investigación [... ] a pesar de los rasgos de irreductible especificidad que cualquier estudio de caso muestra.

Esta metodología nos permite desarrollar los múltiples conocimientos sobre lo que sucede en la educación desde el punto de vista de los propios sujetos estudiados, utilizando los documentos escritos o hablados, lo cual nos permite mirar lo más íntimo de los procesos educativos.

Por ello, estimamos la elección de esta metodología, pues somos conscientes de la importancia de conocer desde la realidad de los centros escolares con respecto al alumnado gay. Asimismo, su realidad cotidiana nos permite ampliar el conocimiento sobre lo que realmente sucede en las aulas a través del punto de vista de las personas implicadas, personas anónimas que aportan, por medio de sus testimonios, una mirada personal e íntima de su proceso educativo.

Hemos realizado una entrevista en profundidad, como instrumento de la investigación, con preguntas abiertas, queriendo dejar a nuestro informante la libertad de profundizar en cada una de las cuestiones. Esto ha suscitado nuevas preguntas y nuevos temas que, sin este instrumento, no podríamos haber abordado. Como apunta Mariezkurrena (2008, p. 231):

El objetivo de una entrevista de historia oral no es obtener «datos», sino entender una vivencia, ya que todo lo que aporta es significativo. Aunque nuestro informante incurra en fallos de memoria, exageraciones o ficciones, todo ello confiere significado a la historia de su vida.

En la primera fase elaboramos una entrevista semiestructurada, resultado de anteriores conversaciones informales con el sujeto de estudio, que nos situó a plantearnos las cuestiones más relevantes. En la entrevista se trasladaron al sujeto de manera organizada y sistematizada, atendiendo a los objetivos planteados, las diferentes preguntas que se habían programado. Esta entrevista ha sido grabada respetando todas las pautas de la ética de la investigación y posteriormente transcrita. En esta línea, consideramos la narrativa como un acto compartido de conocimiento, reflexión y aprendizaje. 


\section{HOMOFOBIA EN LA ESCUELA: PSICOLOGÍA AFIRMATIVA GAY EN EL CONTEXTO EDUCATIVO}

\section{RESULTADOS ALCANZADOS}

Una vez finalizada la entrevista y habiendo transcrito y analizado pormenorizadamente las vivencias de nuestro informante, pasamos a detallar la realidad descrita por él mismo.

En primer lugar, al tratarse de una persona que ha finalizado su etapa primaria y secundaria (tanto secundaria obligatoria como bachillerato), en aras de realizar un análisis estructurado, se expondrá los resultados de forma secuencial y diferenciada por etapas educativas.

Nuestro informante comienza relatando una de sus primeras experiencias que recuerda donde sufrió homofobia:

"Cuando entré en el colegio, recuerdo que yo era muy pequeño, en el patio unos niños mayores me dijeron que yo hablaba y andaba como una niña y se rieron. De verdad que no sabía qué querían decir pero claro, se rieron y yo pasé mucha vergüenza..."

Aquí podemos observar como desde una edad muy temprana se puede sufrir homofobia y cómo este tipo de acoso escolar no está restringido a edades más avanzadas como la adolescencia.

"En 5ํurso, en educación física los niños no querían estar en mi equipo y me decían mariquita. Pensé incluso en dejar de ir al colegio para siempre, no quería estudiar. Yo se lo dije al profesor y él me dijo que tampoco era para tanto y ni me miró..."

A través de esta experiencia observamos el aislamiento que sufre por parte del grupo de iguales no solo durante la hora del recreo donde la supervisión adulta se hace más complicada sino que ocurre durante el desarrollo de una clase. Observamos cómo el alumno se dirigió a un docente para exponer la situación que estaba viviendo en el colegio, pero éste no ofreció ayuda para solventar el problema, no se mostró colaborador ni empático para entender el sufrimiento que le estaban expresando el alumno. Por lo tanto no se tomó ninguna medida de protección para nuestro informante. Como consecuencia de la desprotección, nuestro informante que hasta esa fecha obtenía buenos resultados académicos y mostraba motivación hacia el ámbito educativo se plantea no acudir a clase y percibe un contexto poco seguro.

"En 5ํㅡㄹ primaria también tuve una excursión a un camping durante 5 días y yo al buscar a gente para dormir en la misma habitación me dijeron que conmigo no porque era maricón, ese día llegué a casa llorando y fue la primera vez que hablé con mi madre y le dije que me estaban diciendo esas cosas, pero tenía tanto miedo al rechazo que le dije que era mentira y que a mi no me gustaban los chicos..."

Nuestro informante expresa el rechazo explícito que de nuevo sufre por parte de sus iguales. Además de esto, debido al miedo y el propio rechazo que él mismo sentía le fue imposible poder expresarlo con su madre. Observamos en este caso homofobia interiorizada en el alumno, ya que muestra comportamientos, actitudes y sentimientos que impide que pueda identificarse con su orientación sexual. Esto tiene como consecuencia el desarrollo de sentimientos de inferioridad y baja autoestima.

Analizando las vivencias en educación secundaria obligatoria, nuestro informante relata:

"Cuando pasé al instituto fue terrible, lo único que pensaba todos los días era que nadie se diera cuenta que era gay y que no se me notara. Pero claro había gente de mi colegio anterior así que en los recreos me llamaban maricón cuando pasaba delante de algún grupo, al final de curso me pasaba todo el recreo en la entrada sin moverme para que nadie me viera y me pudiera insultar".

Seguimos observando cómo nuestro informante sigue mostrando homofobia interiorizada en esta etapa educativa y concibe la homosexualidad como un aspecto de sí mismo negativo y que merece ser ocultado. Para ello intentó no mostrar aquellas actitudes y comportamiento femeninos, que socialmente han estado reservados para las mujeres. Como consecuencia del miedo al rechazo, el alumno se aisló socialmente. Optó por preferir estar solo, de esa forma se sentía protegido ante las posibles amenazas y daños que el entorno educativo le pudiera ocasionar. Se trata de una respuesta compatible con personas que han sufrido un acontecimiento traumático.

No obstante, nuestro informante refiere que siempre ha mostrado interés por pertenecer a un grupo de iguales y tener relaciones sociales cercanas y de confianza; y que durante toda su etapa educativa ha intentado encajar 
en los grupos de chicos aunque sin mucho éxito. No obstante, ha notado una mejor conexión con las chicas ya que éstas no lo insultaban o denigran. Lo cual le ayudó para poder expresar lo que sentía con una de ellas:

"En 2ํㅡㅇ, le dije a una amiga que me gustaban los chicos y que me guardara el secreto. Se lo conté en el recreo, y después hablamos con mi tutora. Se lo dije porque llevaba dos semanas sin poder ir a clase porque me encontraba muy mal emocionalmente y el médico me dijo que era ansiedad y entonces como me encontraba tan mal empecé a ir a un psicólogo pues necesitaba ayuda...".

Observamos que tras hablar con su amiga y ésta normalizara y aceptara lo que estaba expresando nuestro informante, éste experimentó en primer lugar la normalización de su expresión afectivo-sexual, lo cual favoreció que pudiera posteriormente hablar por primera vez sobre su homosexualidad con un adulto.

No obstante, seguimos observando las consecuencias del bullying homofóbico que ha venido sufriendo a lo largo de estos años, a través de la aparición de sintomatología ansiosa. A raíz de este suceso comienza a realizar sesiones de psicoterapia basada en Psicología Afirmativa Gay y empieza a trabajar tanto la sintomatología anteriormente descrita como la homofobia interiorizada, entre otros aspectos.

A raíz del proceso de psicoterapia pudo afrontar las relaciones sociales sin miedo y aprendió a pedir ayuda cuando sintiera que estaba en una situación de peligro tanto a su familia como amigos e incluso con las fuerzas de seguridad. Pudo igualmente establecer relaciones sociales satisfactorias con personas con las que se identificaba, así como conocer a referentes no heteronormativos. Todo ello tuvo como consecuencia una mejor aceptación de sí mismo y ofreció la posibilidad de expresarse tal y como él es:

"En 4 ya lo sabían todos y la verdad que cuando lo conté, en la clase mucha gente se acercó a decirme que era valiente, me sentía más seguro a partir de ese momento..."

También en 4ํE:

"Pero también en ese curso me abrí una cuenta de instagram porque me sentía más seguro de mí mismo, y empecé a recibir mensajes anónimos, me decían de todo, maricon, enfermo, incluso me Ilamaron pederasta. La verdad que había días que Iloraba bastante, porque no entendía tanto odio, no tenía ganas de nada, empecé a sacar malas notas, a no querer ir al colegio..."

Observamos de nuevo las consecuencias a nivel psicológico con motivo de estas experiencias traumatizantes. Incidiendo de forma adversa en la percepción negativa de la homosexualidad (homofobia interiorizada) y en una baja autoestima. Llegando incluso a pensar de nuevo en abandonar los estudios. En este caso nuestro informante vuelve a aislarse tanto de su grupo de amigos como de su familia, mostrando una mayor sensibilidad y baja tolerancia a la frustración en otros ámbitos de su vida. En ese momento necesitó que se intensificaran las sesiones de psicoterapia.

Durante los años de bachillerato:

Debido a la experiencia negativa en el centro donde realizó los estudios tanto de primaria como secundaria, nuestro informante junto a su familia realizaron una búsqueda de centros de educación donde se ofertara bachiIlerato y tuvieran una perspectiva LGBTIQA+:

"En 1ํ de bachillerato me cambié de colegio, me dijeron que fuera a uno donde los profesores eran muy abiertos y guays. La verdad que cuando entré el primer día vi en mi misma clase gente super diferente entre ellos y eso me relajó. Era otro ambiente muy distinto, e incluso un profesor dijo que estaba casado con otro hombre y eso me ayudó bastante, en este instituto el profesorado me apoyaba"

Se observa la naturalidad con la que se expresa y respeta en el aula la diversidad. Nuestro informante percibe por primera vez un entorno académico seguro y libre de homofobia. Esto le permitió poder seguir desarrollando su identidad y la expresión de género con la que se identificaba:

"En el verano de 1ํ a 2- decidí que quería vestir con tacones y faldas algunos días porque me apetecía y me veía más guapo. Cuando empecé las clases creía que no iba a poder pero los compañeros de mi clase me dijeron que no podía dejar de ser yo. Los primeros días iba con miedo a clase, sentía que en cualquier momento iba a pasar lo mismo que me pasaba antes y que yo me lo estaba buscando solito. Tenía hasta pesadillas muchas noches" 


\section{HOMOFOBIA EN LA ESCUELA: PSICOLOGÍA AFIRMATIVA GAY EN EL CONTEXTO EDUCATIVO}

De nuevo seguimos observando las consecuencias del bullying una vez incluso no existe tal acoso escolar. Observamos sintomatología compatible con trastorno de estrés postraumático.

"Un día recuerdo que un chico en la puerta del colegio me insultó, haciendo referencia a que parecía una mujer, y lo hablé en clase y se enteró la profesora. Al día siguiente localizamos en la entrada al chico y se habló con él y tuvo que pedirme disculpas, y hablaron con sus padres en tutoría, a partir de este incidente, en clase la profesora que era muy "apañá", se dieron varias charlas sobre la diversidad sexual"

Nuestro informante relata que tras este suceso se reunió el claustro de profesores y desde el centro tomaron medidas con respecto al alumno que había llevado a cabo ese insulto. El equipo docente determinó la necesidad de llevar a cabo un programa educativo de diversidad afectivo-sexual desde la perspectiva de la psicología afirmativa LGTBI, que fue llevado a cabo en todas las clases del centro educativo. De igual forma todo el profesorado también acudió a dicha formación.

Tanto el proceso de psicoterapia como las medidas que se llevaron a cabo en el centro educativo tuvieron como resultado un cambio positivo en nuestro informante:

"Ahora me siento mucho más libre y en gran parte ha sido por los últimos años de clase. Que me permitieran ser yo mismo y que se me respetara en los últimos años. También el acompañamiento tuyo (haciendo referencia a psicólogo) hace que pueda continuar mis estudios empoderado y creyendo en mí mismo, la terapia me ha venido muy bien, porque me ha dado claridad sobre quién soy y herramientas para seguir adelante y empezar la universidad sin miedos".

Nuestro informante expresa las consecuencias positivas que han tenido las medidas llevadas a cabo. Por un lado ha disminuido la sintomatología compatible con trastorno estrés postraumático, y por otro lado tuvo como resultado la eliminación de la homofobia interiorizada así como el desarrollo de habilidades sociales, el aumento de la autoestima y la eliminación de síntomas depresivos:

"me ha venido muy bien sentirme escuchado y apoyado por un psicólogo especialista temas LGTB, porque me he sentido comprendido y apoyado para seguir en el instituto y no abandonar los estudios, por que..... más de una vez lo había pensado, la verdad que era una idea que desde primaria tenia, dejar el colegio para dejar de sufrir, y ahora tengo muchas ganas de estudiar en la universidad"

No obstante, a pesar del avance conseguido, debido a las secuelas del bullying homofóbico y de las posibles situaciones adversas que puedan ocurrir, continuará en seguimiento terapéutico hasta remisión completa de la sintomatología presente.

\section{DISCUSIÓN}

A continuación, pasaremos a dar sentido a la información recabada y transmitida por nuestro informante para interpretar los múltiples significados de sus vivencias en la escuela. Pretendemos explicar los resultados que se han vertido en nuestra investigación cualitativa. El protagonista de la entrevista, proyecta una visión de una situación de homofobia en el entorno escolar, la cual le ha generado muchas inseguridades y miedos. Este continuo hostigamiento en el entorno escolar, ha incidido directamente en el deterioro de su salud mental. Diversas investigaciones realizadas por Meyer $(2003,2007)$, apuntan que debido al mayor estrés al que están expuestas las personas con orientaciones afectivo-sexuales no normativas la prevalencia de los trastornos mentales será mayor.

Además, nos comenta que se ha sentido muy solo por parte de sus compañeros en la escuela, principalmente en la etapa de Educación Infantil y Educación Primaria, el aislamiento es uno de los efectos directos del bullying homofóbico. La estigmatización que sufren por ser 0 ser percibido las personas LGBTI tiene consecuencias sociales tales como el rechazo, la falta de aceptación, y la falta de apoyo social se asocia directamente con la ansiedad y la depresión (Rivers, y D'Augelli, 2001). Nuestro informante revela que en varias ocasiones ha deseado abandonar los estudios, se detecta una de las repercusiones directas del bullying homofóbico en el bajo rendimiento escolar. 
En sus palabras, se refleja que desde las primeras etapas educativas ha sufrido constantemente insultos homofóbicos, frente a la impasibilidad de sus compañeros y lo más grave del propio profesorado. La escasa intervención por parte del profesorado y negación de la situación de bullying homofóbico provoca en nuestro informante mayor vulnerabilidad y desamparo: "Los estudios apuntan que, en muchas ocasiones, el profesorado no contribuye a erradicar el problema, sino a agravar" (Cerezo, 2015, p.424). Consideramos que la intervención directa y rápida del profesorado en los casos de bullying homofóbico es clave y necesaria para solucionar estas situaciones. Autores como Elsbree (2002), resaltan como la formación del profesorado en la diversidad sexogenérica es un factor determinante en la inclusión.

Además, debido a la constante evolución de la tecnología, se refleja que también ha sufrido ciberbullying homofóbico, autores como Herrera et al. (2018), afirman que el ciberbullying produce en las víctimas diversos efectos negativos como son el bajo rendimiento académico, el absentismo escolar y la depresión entre otros.

Se revela en sus palabras como el cambio en la etapa de secundaria a un centro más inclusivo, supuso una mejora, el apoyo tanto del profesorado como de las compañeras y compañeros de clase es un factor positivo para su inclusión educativa. Es interesante resaltar la importancia de tener referentes gay como uno de sus profesores, para el proceso de inclusión del alumno. Como señalan Sánchez y Mestre : "la necesidad de incluir en el currículum escolar de forma explícita la diversidad sexo-genérica, pues facilitaría los procesos de la inclusión educativa"(2021, p.6). Asimismo, en este centro, cuando fue insultado, se tomaron medidas de manera directa y tuvo una repercusión positiva tanto para nuestro protagonista como para toda la clase.

A lo largo de la entrevista, se vislumbra el papel clave que ha desempeñado la psicología afirmativa gay en la mejora y acompañamiento de nuestro protagonista en las diversas etapas educativas. Mediante el acompañamiento psicológico, se han superado traumas, se han adquirido diversas habilidades sociales y estrategias para enfrentar el bullying homofóbico. El apoyo psicológico recibido ha sido determinante en la mejora y el proceso inclusivo en el ámbito educativo y superación de los traumas causados por el bullying, gracias a la terapia nuestro informante puede enfrentar con herramientas la entrada en la Universidad.

\section{CONCLUSIONES}

Tras la entrevista con nuestro informante hemos podido recabar una importante información acerca de las experiencias homófobas vivenciadas en el ámbito educativo. La Psicología Afirmativa Gay ofrece un marco de referencia respetuoso con la persona que expresa sus vivencias. En primer lugar, es importante hacer referencia como a través de la psicoeducación afectivo-sexual que recibe nuestro informante se ofrece al mismo una visión completa y compleja sobre dichos aspectos, lo cual favorece considerablemente la reducción de la estigmatización y de la homofobia interiorizada.

A su vez, la expresión y eliminación de la homofobia internalizada ha sido fundamental para el desarrollo afectivo-sexual saludable de nuestro informante. La terapia cognitivo-conductual se ha usado para tratar y trabajar las distorsiones cognitivas como por ejemplo el miedo al rechazo o las creencias asociadas al aislamiento social.

Por otro lado, el acompañamiento terapéutico basado en la Psicología Afirmativa Gay permite a la persona poder, entre otras cosas, expresar en un entorno seguro las experiencias traumáticas vivencias como consecuencia de la homofobia. Para ello se ha llevado a cabo mediante la terapia EMDR (siglas en inglés, Desensibilización y Reprocesamiento por los Movimientos Oculares). La cual se encuentra avalada por la Organización Mundial de la Salud para el tratamiento del trauma. En cuanto a la presencia de ansiedad, ésta se redujo hasta niveles normativos tras el abordaje de la realidad afectivo-sexual de nuestro informante como persona homosexual. Se hace referencia con ello a aspectos tales como las relaciones afectivas y amorosas o el miedo al contagio VIH.

De igual forma, es inevitable después de escuchar la experiencia educativa de nuestro informante, como refleja la problemática relativa a la insuficiente formación del profesorado para afrontar de manera inclusiva las diversidades sexo-genéricas. En esta línea, Ilegamos a la conclusión de la necesidad de formación tanto inicial como continua del profesorado en diversidad sexo-genérica. A tenor de lo expuesto, desde los grados de forma- 


\section{HOMOFOBIA EN LA ESCUELA: PSICOLOGÍA AFIRMATIVA GAY EN EL CONTEXTO EDUCATIVO}

ción del profesorado se debe contemplar en sus planes formativos la temática de la diversidad sexo-genérica para construir una escuela libre de homofobia. Además de una actualización didáctica del profesorado sobre la inclusión del alumnado gay en los centros escolares. Se constata la importancia de la formación del profesorado para Ilevar a cabo la inclusión educativa del alumnado gay y prevenir e intervenir si hubiese casos de bullying homofóbico.

Esta investigación refleja la necesidad de establecer estrategias de prevención frente al bullying homofóbico, como incluir en el currículum escolar de forma explícita la diversidad sexo-genérica, pues facilitaría los procesos de la inclusión educativa. Al igual que lograr la sistematización de estos contenidos para que no sean tratados de forma puntual, y trabajar de manera inclusiva la heterosexualidad y las sexualidades no normativas en las diversas etapas educativas.

A modo de conclusión, se demuestra la necesidad del asesoramiento psicológico afirmativo gay en los procesos de bullying homofóbico en la escuela. Esto nos lleva a la reflexión de la necesidad dentro del ámbito escolar de la atención psicológica afirmativa gay desde el propio centro. Por todo ello, para comenzar a dibujar una escuela libre de homofobia, es necesario que en los centros escolares se atienda a las necesidades tanto educativas como psicológicas del alumnado gay desde las primeras etapas de la educación.

Consideramos necesario el trabajo psicoeducativo conjunto, entre el profesorado y el equipo psicológico del centro en casos de bullying homofóbico, en continuo diálogo con los familiares. El trabajo de todos los miembros de la comunidad educativa es clave para erradicar los casos de bullying homofóbico en la escuela. Pero valoramos que se debe hacer hincapié en la prevención, para construir una escuela verdaderamente inclusiva con todo su alumnado, libre del acoso homofóbico. En esta línea el enfoque inclusivo debe impregnar todos los elementos que configuran el currículum educativo y a toda la comunidad educativa.

\section{REFERENCIAS BIBLIOGRÁFICAS}

Abad Miguélez, B. (2016). Investigación social cualitativa y dilemas éticos: de la ética vacía a la ética situada. EMPIRIA. Revista de Metodología de las Ciencias Sociales, 34, 101-119.

Agustín, S. (2009). Diversidad sexual en las aulas. Evitar el bullying o acoso homofóbico. Badajoz: Fundación Triángulo y Plural.

American Psychology Association (2012). Guidelines for psychological Practice with Lesbian, gay and bisexual clients. American psychologist, 67 (1), 10-12

Ardila, R. (2007). Terapia afirmativa para homosexuales y lesbianas. Revista Colombiana de Psiquiatría. 36 (1), 67-77.

Berná, D., Cascone, M. y Platero, R. (L.) (2012). ¿Qué puede aportar una mirada Queer a la educación? Un estado de la cuestión sobre los estudios sobre la LGTBfobia y educación en el Estado Español. Scientific Journal of Humanistic Studies, 4(6), 1-1.

Blais, M., Gervais, J., y Hébert, M. (2014). Internalized homophobia as a partial mediator between homophobic bullying and self-esteem among youths of sexual minorities in Quebec (Canadá). Ciencia e Saude Coletiva, 19(3), 727-735.doi:10.1590/1413-81232014193.16082013

Borges, K. (2009). Terapia afirmativa: uma introdução á psicologia e á psicoterapia dirigida a gays, lésbicas e bissexuais. Edicoes GLS.

Boza, A., Méndez, J.M., Monescillo, M. y Toscano, M.0. (Coords.) (2010). Educación, Investigación y Desarrollo Social. Narcea.

Bromseth, J. (2002). Public places-public activities? Methodological approaches and ethical dilemmas in research on computer-mediated communication contexts, Researching ICTs in Context, 33-62.

Carrer y Cifuentes (2019). L'LGTBI-fòbia entre infants i adolescents. Una problemàtica social a resoldre. Dossier Catalunya Social. Propostes des del tercer sector. Num. 58.

Cerezo Ramírez, F. (2015). Intimidación homofóbico. El papel del profesorado. Revista Internacional de Psicología 
del Desarrollo y la Educación, 1 (1), 417-424.

COGAM. (2016). Ciberbullying LGBT-fóbico. Nuevas formas de intolerancia. Grupo de Educación de COGAM.

Elsbree, A. (2002). Disruptive Pedagogies: How Teacher Educators DisruptHomophobia. University of Wisconsin.

D Augelli, A. R., Pilkington, N. W., Hershberger, S. L. (2002). Incidence and Mental Health Impact of Sexual Orientation Victimization of Lesbian, Gay, and Bisexual Youths in High School. School Psychology Quarterly, 17(2), 148 -167. https://doi. org/10.1521/scpq.17.2.148.20854

Espelage, D. L., Aragon, S. R., Birk ertt, M. (2008). Homophobic teasing, psychological outcomes, and sexual orientation among high school students. What influence do parents and schools have? School Psychology Review, 37, 202-216.

Foucault, M. (1976). Vigilar y castigar. Siglo XXI Editores.

Garrido, R., Morales, Z. (2014). Una aproximación a la Homofobia desde la Psicología. Propuestas de Intervención. Psicología, Conocimiento y Sociedad, 4(1), 90-115.

Hadler, M. (2012). The influence of world societal forces on social tolerance. A time comparative study of prejudices in 32 Countries. The Sociological Quarterly, 53(2), 211-237.

Herrera, M., Romera, E., y Ortega-Ruiz, R. (2018) Bullying y cyberbullying en latinoamérica. Un estudio bibliométrico. Revista Mexicana de Investigación Educativa, 23(76), 125-155.

Martxueta A y Etxeberria J. (2014). Análisis Diferencial Retrospectivo De Las Variables De Salud Mental En Lesbianas, Gais Y Bisexuales (Lgb) Víctimas De Bullying Homofóbico En La Escuela. Revista de Psicopatología y Psicología Clínica, 19,(1), 23-35.

Mariezkurrena Iturmendi, D. (2008). La historia oral como método de investigación histórica. Gerónimo de Uztariz.

Massot, I. (2003). Jóvenes entre culturas: La construcción de la identidad en contextos multiculturales. Desclèe de Brouwer.

Meyer, I. H. (2003). Prejudice, social stress and mental health in lesbian, gay and bisexual populations: Conceptual issues and research evidence. Psychological Bulletin, 129, 674-697.

Meyer, I. H. (2007). Prejudice and discrimination as social stressors. En I. H. Meyer \& M. E. Northridge (Eds.), The health of sexual minorities: Public health perspectives on lesbian, gay, bisexual and transgender populations (pp. 242-267). Nueva York: Spring

Pichardo, J.I. (2012). El estigma hacia personas lesbianas, gays, bisexuales y transexuales. En E. Gaviria, C. García, F. Molero (Coords.): Investigación-acción. Aportaciones de la investigación a la reducción del estigma (pp. 111-125). Madrid: Sanz y Torres.

Pichardo, J.I., De Stéfano, M., Faure, J., Sáenz, M. y Williams, J. (2015). Abrazar la diversidad: propuestas para una educación libre de acoso homofóbico y transfóbico. Madrid: Ministerio de Sanidad, Servicios Sociales e lgualdad.

Platero, L. y Gómez, E. (2007). Herramientas para combatir el bullying homofóbico. Talasa.

Pujadas, J. J. (2000). El método biográfico y los géneros de la memoria. Revista de Antropología Social, (9), 27158.

Quiceno, J. M., Mateus, J., Cardenas M., Villareal, D. y Vinaccia, S. (2013). Calidad de vida, resiliencia e ideación suicida en adolescentes víctimas de abuso sexual. Revista de Psicopatología y Psicología Clínica, 18, 107117.

Rivers, I., \& D'Augelli, A. R. (2001). The victimization of lesbian, gay, and bisexual youths: Implications for intervention. En A. R. D'Augelli \& C. J. Patterson (Eds.), Lesbian, gay, and youths: psychological perspectives (pp. 199-223). Nueva York: Oxford University Press.

Rivers I. (2000). Social exclusion, absenteeism, and sexual minority youth. Support for Learning, 5, 13-18.

Rivers, I. (2004). Recollections of bullying at school and their long-term implications for lesbians, gay men, and 


\section{HOMOFOBIA EN LA ESCUELA: PSICOLOGÍA AFIRMATIVA GAY EN EL CONTEXTO EDUCATIVO}

bisexuals. Crisis, 25(4), 169-175.

Rivers, I., Cowie, H (2006) Bullying and homophobia at UK schools: A perspective on factors affecting resilience and recovery. Journal of Gay and Lesbian Issues in Education, 3, 11-43.

Sánchez Torrejón, B. (2021). La formación del profesorado de Educación Primaria en diversidad sexo-genérica. Revista Electrónica Interuniversitaria de Formación del Profesorado, 24(1). https://doi.org/10.6018/reifop.393781

Sánchez Torrejón, B. \& Mestre Malfran, Y. (2021). Ciberbulying homofóbico en Educación Primaria: hacia una escuela libre de homofobia. Hachetetepé. Revista cientifica de Educación y Comunicación, (23), 1-11.

Takács, J. (2006). Social exclusion of young lesbian, gay, bisexual and transgenderpeople (LGBT) in Europe. ILGA Europe.

Vargas L, Flisser A, Kawa S. (2007). Consentimiento informado. En: Perez Tamayo R, Lisker R, Tapia R, editors. La construction de la bioetica. pp. 119-34. Mexico: FCE;

Wiles, R., Crow, G., Heath, S., y Charles, V. (2006). Anonymity and confidentiality, Conference ESRC Research Methods Festival. University of Oxford. 